\title{
What is Time in Some Modern Physics Theories: Interpretation Problems
}

Ivan A. Karpenko

National Research University Higher School of Economics, School of Philosophy, Laboratory for Philosophical Studies, Moscow, Russia

e-mail:gobzev@yandex.ru

\section{Abstract:}

The article deals with the problem of time in the context of several theories of modern physics. This fundamental concept inevitably arises in physical theories, but so far there is no adequate description of it in the philosophy of science. In the theory of relativity, quantum field theory, Standard Model of particle physics, theory of loop quantum gravity, superstring theory and other most recent theories the idea of time is shown explicitly or not. Sometimes, such as in the special theory of relativity, it plays a significant role and sometimes it does not. But anyway it exists and is implied by the content of the theory, which in some cases directly includes its mathematical tools. Fundamental difference of space-time processes in microcosm and macrocosm is of particular importance for solving the problem. In this regard, a need to understand the time in the way it appears in modern physics, to describe it in the language of philosophy arises (satisfactory for time description mathematical tools also do not exist). This will give an opportunity to get closer to the answer on question of time characteristics. And even if we do not obtain the exact answer, we will still be able to formulate the right question about its nature. For this purpose, the present research carries out analysis of the key theories of modern physics with regard to historical and scientific, historical and philosophical perspectives. In some cases, this gives an opportunity to detect the succession of the associated with time perception ideas, their development, as well as the origination of fundamentally new ones. During the analysis, the correct characteristics of time are formulated from the point of view of physical theory and the attempt to state the nature of time is made. On the ground of conducted research, the conclusions about current state of the problem and its future solution perspectives are drawn.

Keywords: time, philosophy of science, modern physics, concept of space and time. 


\section{Introduction}

The problem of time is not an entirely physical problem. Physics itself does not contain a "time theory". In the sense that there have not been made any direct attempts to find the natural-science answer to what is the time. Nevertheless, the concept of time emerges in science anyway and still requires an explanation. Time fulfills an important role in physics of XX and XXI centuries, though often a hidden role. This applies to both theories of macrocosm and theories of microcosm. In the theory of relativity time is established as a secondary feature, a derivative of the velocity and mass, but nevertheless, it exists (although as an illusion) and evokes the need of its philosophical interpretation. In quantum field theory time also (though implicitly) occurs in relation to the interpretation of the experiment results - for example, "where the particle was before observation of it". This "before" exactly indicates the presence of time (more precisely, the observer's perception of its presence).

In further theories, which have been the attempts to solve the problem of incompatibility of general relativity theory and quantum mechanics, such as the theory of loop quantum gravity, superstring theory, Shape Dynamics and others, time also fulfills a definite role and evokes the question of its explanation in the frameworks of these theories again. I.e. what meaning of this "time" term has been chosen to be used?

This article deals with the problem of time in the context of several theories of modern physics. In particular, it attempts to answer the question of what the time is in relation to the philosophy of physics (physics itself does not have such an answer). During the research the historical and philosophical practice of terms interpretation has been reviewed.

\section{Problem Statement}

As noted by Gerald James Whitrow, the author of ones of the fundamental works on time matter [45], [46], [47], time geometrization is basically typical for physics. Albert Einstein has noted the same [12, p. 141]. If we have got used to conceive space with coordinate system, in terms of dimensions and distances ${ }^{1}$, then there are no specific temporal concepts, which would characterize time itself. To some extent, this explains the difficulty in interpretation of various physics theories usage of time concept - it is hard to interpret something that has no endemic characteristics. Because of this exact absence of its essential attributes time has become the subject for geometrization. This becomes particularly obvious in the theory of relativity and in the description of time in Minkowski space (constructed on the basis of Henri Poincaré [39, pp. 129-176] and Einstein [11, pp. 891-921] works by Hermann Minkowski [34,pp. 75-88]), where time constitutes the fourth coordinate and products light velocity and event time. In general, in the theory of relativity the problem is solved radically - time has been almost eliminated, it is nothing more than an illusion of perception. However, in this case the situation is the same not easy and time rejection causes serious difficulties with universe evolution (these difficulties are to be discussed later).

The traditional time discourse creates purely spatial questions: if time is discrete or continuous, whether it can be identified with change (sequence of events) and duration, whether it is objective or subjective, relative or absolute, fundamental or not, etc. These issues result from the classical intellectual intuition, revealed back at works of Zeno, then Aristotle, the Stoics and later Augustine. That intuition has been also noteworthy preserved almost unaltered at Galileo Galilei and Isaac Newton works.

It is significant that in these cases, where peculiarity of time is stated (ones of the latest works, as an example, Lee Smolin books [42],[32], which proves the fundamental nature of time and its independence of spatial terms), time definition still remains unclear.

As a consequence, the most important question on time matter seems to be the following: does time even exist? The present research certainly does not contemplate on giving an ultimate 
answer. However, the analysis of the time concept and its possible characteristics, emerging in modern physical theories, allows close approaching to the answer.

\section{Brief Historical Background of the Problem}

Concerning discreteness and continuity the problem of time was, apparently, first described by Zeno of Elea ${ }^{2}$ in his famous paradoxes. The Achilles, Dichotomy, Arrow and Stadium paradoxes are aimed against motion. The first two of them deny motion, if space and time are viewed as continuous, the latter two, when both space and time are discrete. Alexander Koyre in Notes on the Zeno's paradoxes [27] has made an important observation that these paradoxes, in fact, are not related to motion and only concern it insofar as the motion takes place in time and space. Then an important question can be formulated: is movement (or any change) possible out of time? We shall return to this matter in the following analysis.

Further, Koyre reveals that all the four arguments allow a double interpretation, which means that the Achilles and Dichotomy paradoxes stay valid if we consider space and time as discrete. Similarly, if we consider the Arrow and Stadium from the point of space and time continuity, the paradoxes still remain unsolvable.

The next step suggests that the concepts of space and time can be ignored and instead the mathematical continuum should be considered, without depriving the paradoxes of their original meaning. After applying such transformations Koyre concluded that the problem is much more intense than the experts and critics of Zeno had imagined and discovered (after paradoxes translation into the language of mathematics) that these paradoxes are rooted in a hidden form in any geometric theorem, in any algebraic formula, in any arithmetic assertion. I.e. the problem is inherent in mathematics and geometry, but within this mathematical approach it ceases to be a problem, since in terms of mathematics there is nothing paradoxical in continuity. It may lead to a conclusion that our traditional intuitive ideas about motion, time and space require serious consideration, only then the paradoxes will cease to be paradoxical. Or (which is quite the same) the paradoxes were generated by misconceptions about movement, space and time.

Another issue, which refers to Zeno's paradoxes, is whether time should actually "flow". Bertrand Russell [41,p. 813] has not seen any contradiction in the arrow seemingly leaping from one position to another. This means that motion in time can be understood as a change of position, following the example of the trotting second hand. Quick change of position is perceived as smooth motion. Viewed this way, motion lacks what we intuitively understand as motion. At any given moment the arrow resides in a new place. However, we can consider this motion.

In Timaeus Plato describes time as a rotating similarity of eternity [37, 37a-38c], apparently, in accordance with the cyclic tradition. Real time is frozen eternity; its similarity is the time of rotating sky, a move from number to number.

The first attempt to scientifically analyze the concept of time might have been made by Aristotle in Physics. He was naturally dissatisfied by Plato's approach, primarily because he could not accept Plato's Ideas as prototypes of the observed reality. According to Aristotle, time and motion are related, but not identical. In his words, "time is the number of motion" $[1, I V, 11]$ and it is continuous. Motion is measured by time, and time is determined by motion. But a necessary condition of motion is space, which means that there is no time without space. Perfect motion is circular (here we again witness the tradition of cyclical time concept, the movement of celestial bodies, the circle of life, the change of seasons, etc.).

Plotinus disagrees with Aristotle. According to him, time (following Plato) is derived from eternity. But he does not perceive time as motion or measure of motion (not the number of motion) [38, III, 7].

His objections towards time as motion or number of motion can be briefly explained as follows: motion presupposes time, but time does not require motion and can fully coincide with quiescence. Which means that "if we assume the possibility of motion out of time, then equating 
time to it makes it even more incomprehensible, as consequently, time would be one thing and motion quite another" [30,p. 442]. So, time is one thing, and motion is another. Also Plotinus shows that since motions can be different, i.e. different distances can be covered in the same period of time, then times must be different too, which is impossible (it is interesting that idea of time relativity appears here by now, derived from motion, but though denied). Consequently, distance cannot be conceived as time. This can be interpreted as a protest against the spatial nature of time.

Later Plotinus claims that time is not a number, arguing that anything can be measured with numbers, not just time, so time is time, and a number is a number. In terms of a definition, according to Plotinus, time is the length of an eternal life of a soul (but again length is a spatial characteristic).

A special place in the time studies is occupied by famous reflections by Augustine. He claims that time is not motion, because there is no real past and no real future, but there is present. However, this present lacks durability; it's momentary, so, in fact, it does not exist. But all the three times - the past, the present and the future - exist in human soul. We wish to interpret this as the statement of illusiveness, subjectivity of time, but Augustine does not explicitly say this. Time still exists and he expresses it in his famous formula, "time exists only because it tends to disappear" [2, 111.XIV.17]. He rather reveals the psychology of time perception - occurring in one's soul an image of the present allows thinking about the past and the future following the model of this present (a kind of induction). Augustine has also introduced a novelty, which can be directly attributed to physics. Discussing a popular issue of that time, "What had God been doing before he created the world?", he boldly declares: nothing. For the simple reason that time has been created together with the world, how can we speak of before and after, if there was no time? Without time these concepts simply do not make sense. Thus Augustine states the following idea: there is no external fundamental eternal time as an arena for physical laws. Time occurs together with the Universe.

In his commentary to Plato's Timaeus Neo-Platonist Proclus (apparently, following Iamblichus's ideas) [40] has developed kind of time and eternity dialectics. Time for him is duration, fluidity, continuity. Time is (again in Plato`s spirit) a motile image of eternity and eternity is a fixed image of time. Time is associated with motion and for its flow something to force every event into motion is required, as each event needs something to cause its movement. The initial cause of motion is eternity.

Damascius has developed these ideas working on the problem of the essence of time [30, pp. 436-439]. But he has introduced the time quantized. If time consists of non-durable moments of the present, it would be impossible to make them up into a proper duration. The same would be adding even an infinite number of non-dimensional pieces - you still get nothing. Thus, the time should consist of indivisible segments of the present, which all have duration. In other words, time leaps. Damascius explains this by giving an example of human thinking: a thought seems to be continuous; however, it cannot contemplate all at once: first, it is aimed at one thing, then at another, and so on. This attitude can be interpreted as an attempt to prove the discreteness of time. Moreover, the velocity of the time stick-slip motions results in the fact that different movements have different time (which was denied by Plotinus). In fact, this means that time is relative and its relativity is determined by the motion velocity (though, obviously, Damascius was guided by completely different grounds for his intuition, compared to the later relativists).

Philosophers and scientists of later times (the Middle Ages and Modern Period) have deepened the already existing ideas or argued with them, but in general, have remained within the same agenda. However, some other views on time that have defined the modern discourse are worth mentioning, meaning the concepts of Kant, Leibniz, Newton and Einstein.

Newton has insisted on the objective status of time. However, he avoids metaphysics, he does not define the time. But he claims it to be absolute. "Absolute, true and mathematical time, of itself and from its own nature flows equably without regard to anything external and by another name is called duration" [35, p. 30]. Time exists and it is duration. While with the aim of proving 
the absolute space Newton has conducted an experiment with a rotating bucket (which, however, proves nothing, as noted by Ernst Mach [31, pp. 198-199]), but to prove the existence of absolute time he has had no reasoned arguments. Gottfried Leibniz, the opponent of Newton, on the contrary, has postulated the relativity of time, deriving it from the principle of sufficient reason and the principle of identity of indiscernible ${ }^{3}$. One can say that he has deepened Augustine's argumentation on the matter of what God had been doing before the he created the world [29, $p$. 56]: without events and things (of the world) there is no time. But this does not mean that Leibniz has denied time; it does exist and, moreover, it is universal (it makes it obvious that there is nothing in common between Leibniz's and Einstein's concepts of time).

Kant has put the objectivity of time under the question once again. It is interesting how Losev makes a rather sharp remark about Kant's idea on apriority of time, arguing that it was entirely borrowed from Plotinus [30, p. 447]. According to Kant, time is an a priori form of sensuality, which enables us to organize the experience of interaction with the world in our perception [21, pp. 56-58]. It is nonobjective, there is no time itself. Therefore, discussions on the nature of time, its essence or properties are meaningless - they must be limited to our perception, to the activity of consciousness ${ }^{4}$.

Developing the relativity concept, Einstein has formulated the last fundamentally new idea about time, which corresponds to the classical intuition (intuition about macrocosm only, to quantum mechanics, for instance, this intuition cannot be applied). The fundamental novelty of his step is the assertion of relativity of simultaneity, where under the same events seem to be differently separated in time for different observers, depending on the movement velocity (including direction) of the last.

\section{The Current State of the Problem}

Each of the mentioned time theories gives rise to specific complexities, which besides originate not from the viewpoint of physics, where utilized mathematical tools on the assumption of consistency ensure adequate descriptions. Difficulties arise while attempting to perform philosophical analysis.

In modern physics the notion of time discreteness is rather popular. This does not anyhow contradict the fact of mathematical time continuity: a mathematical theory that would virtually explain time does not exist. Therefore, following the ideas of Russell and Zeno, one can assume that a time quantum, "chronon", is a Planckian quantity. Upon this assumption, however, two issues arise. The first and the most obvious issue is the debatable representation of a time unit as a quantum, i.e. something that has fixed dimensions. In this case time appears to be just a particular spatial dimension, where the specific movement takes place. Thereat one can say that between point (event) $\mathrm{A}$ and point (event) $\mathrm{B}$ there is a certain number of time quanta. In theory, there is no paradox here - movement in time can be represented as a saltatory (quantized) motion in quiescent state at the maximum permissible velocity. That is, when something is stationary in a point of threedimensional space it can be interpreted within the STR in such a way that it moves at the velocity of light in time and, therefore, moves in the space, because the space changes over time run.

This raises another problem. If time is quantized, what do the changes of space in time mean? Does the time exist in the "gaps" between time quanta? If it does, we would have to admit the existence of space out of the time intervals, which seems absurd, because it would make us admit another kind of time and so on, and so forth, which leads us to a vicious circle. Instead, if time does not exist in the "gaps", the world is created all over again at every moment, which also contradicts our intellectual intuition ${ }^{5}$.

As an alternative to divisible and infinitely divisible time may serve absolutely indivisible time. It is not quite clear what it means and how we could describe the past and the future under this concept. Probably, this is a representation of time as a single moment (meaning that everything exists simultaneously, but in this case it's unclear why we would even need the category of time after all). The concept with more apprehensible continuity at least presupposes durability. 
Durability, as a characteristic of time, is in close relation to the notion of locality. In classical physics, starting with the works by Galileo, Newton and up to Einstein, time is described as local. This basically corresponds to our intuition. In general, the concept of locality concerns space, of course. Its main idea is that in order to get from point A to point B it is necessary to cover a certain distance. However, since the maximum possible velocity is finite (the velocity of light), locality implies the need to spend certain time to cover any distance. It is interesting that Newton's universe is not entirely local, contrary to common belief - in his theory gravity extends instantaneously. In the relativity theory gravity has a fixed velocity - the velocity of light.

In quantum mechanics the non-locality appears (surprisingly, it was established again by Einstein [13, pp. 777-780], who believed that this way he would demonstrate the insufficiency of the quantum theory). Non-locality means that in order to get from point A to point B photons do not have to cover any distance, they immediately reappear at point $\mathrm{B}$, therefore it takes no time. A broader understanding of the non-locality [33], [9] presumes that there are non-local connections between elementary particles in the Universe and the more connections are there, the more strongly marked are the other dimensions. In other words, if in order to relocate macroobjects in addition to the three dimensions one or many more dimensions were discovered, it would mean the presence of non-locality. It is a curious crossover between quantum mechanics and the relativity theory. Within the relativity theory, as previously noted, there is no time itself, but there is space-time. Thus, every motion is a motion in space-time. If we assume the existence of extra dimensions (which, incidentally, Einstein has already tried on the basis of the Kaluza-Klein theory [20, pp. 966-972], [26, pp. 895-906]) and add the quantum-mechanical non-locality, it turns out that non-locality means movement in other dimensions ${ }^{6}$. Experiments to prove non-locality (quantum teleportation) have already been carried out repeatedly with the quite recent last one [8, pp. 775-778]. However, interpretation of their results is a big challenge. On the basis of them, it is possible to conclude that the concepts of distance (as a characteristic of space) and continuity (as a characteristic of time) are invalid, as they are only a matter of our perception. Furthermore, there is only one place where everything happens (if only we can talk about a place here). However, quantum teleportation involves the creation of a duplicate of the original object somewhere else. The presence of this other place immediately undermines the idea of illusory distance. Otherwise, we should assume that this is not a different place, but the same one; and the photon is exactly the same; there are not two of them, but one. But then the question arises: did the teleportation happen? What kind of manipulations have the experimenters performed if nothing has changed compared to the original state? The idea that the object is the same one is consistent with the Leibniz's identity of indiscernible principle.

The problem becomes irrelevant within Newton's absolute space (and Einstein's space ${ }^{7}$ ). It's not necessary to consider that non-locality means being in one and the same place, it is all about photons which instantly share information across any distance. Yes, the photons are indiscernible, but Leibniz's principle remains inviolate because the various positions in space are also a characteristic of a photon (although, in this case, the function of space is uncertain, it becomes even more artificial than the famous ether of the old physics, which at least was required for the propagation of light waves with a finite velocity). But time is eliminated completely. Thus, combining the relative and quantum interpretations of non-locality shows that time does not exist and it is pointless to talk about any of its characteristics, features or properties. For more information on non-locality and the measurement problem, see [24], [22, pp. 16-28].

It is possible to view time as a sequence of events. Only in modern physics processes at the microscopic level are called events. For example, as a first event you can take the first fluctuation or decay of a particle which has launched a chain reaction of events, some of which are cause-related, which is called time (in this case the beginning of time is not necessarily connected with the unique creation). This raises objections (also not new) that time is still not a sequence of events, because the events are still occurring in time, not the time in events (or time is not motion, but motion takes place in time). 
We shall enlarge upon the issue of the "flow" of time. The relativity theory Universe is often called "block" universe, meaning a single space-time in which there is, indeed, no time. The block can be "cut" under different angles (the velocity and direction of motion corresponds to "cutting") this defines the relativity of simultaneity. For different observers different events will be perceived as happening in a different temporal order; what happened earlier for some observers, happened later for others. The order of events is unchanged only within the boundaries of a single light cone. Events beyond the one cone may not be causally related. Here the key word is "to be perceived", as within a block Universe all events are set up, they occur, and our perception depending on certain conditions allows us to notice them in a certain order. This model excludes the flow of time, the sequence of events cannot be called time either, therefore it lacks continuity as well.

Such circumstances are not accepted by as completely satisfactory. The reason is the presence of so-called "arrow of time". Its specificity is that no matter where and how the observers move, they will observe its one and only direction - from the past to the future (in the sense that the past is different from the future). Although, the laws of physics are reversible in time, reality is irreversible. Order turns into chaos and the opposite is very seldom (though this must occur with the same frequency). This is the well-known second law of thermodynamics and its implications, first described by Rudolf Clausius [10, pp. 481-506], and studied by Ludwig Boltzmann [7]. Entropy always increases. Even if self-organization, the growth of the order take place, it presumes the use of energy and the spent sufficient energy leads to the release of insufficient energy (heat), and entropy (as a measure of disorder) is always greater than the increase of the order.

In this case it is important that the second law of thermodynamics might be considered as an evidence of the time flow or, more boldly, as a description of time itself. In this context time could be comprehended as a transition from less probable states to more probable - and the most probable state is the state of equilibrium. However, in this case we would have to admit that at the moment of equilibrium time stops. This is obviously not true, because in any state of equilibrium fluctuations occur, reducing the entropy in the area around them and then again it leads to a decrease in the order degree. Thus, the second law is not time, but it works in time (in the words of Aristotle, motion in time). It points at the arrow of time.

The arrow of time presupposes asymmetry of the universe in time: if the past is fundamentally different from the future, there must have been some special initial conditions. The choice of initial conditions (in the inflation model, for example) is random to a great extent. The point is that knowing the current state of the universe, it is impossible to reconstruct its original state; it could have reached its present state in many different ways. An important role in the history is played by random events (nondeterminate somehow) - fluctuations. Therefore, the choice of initial conditions is large enough. And even if we ever discover what they were exactly, the question of why they were such and not other, since those other might have been, will remain. In fact, the "accidentally" answer is always possible (which does not withdrawn the question "why is an accident possible?")

Another problem is that the recognition of the arrow of time implies the choice of initial conditions with a high degree of order. If the most probable state is equilibrium, then for something that we are observing at the moment the initial state must have been non-equilibrium. Or it was an equilibrium, but from time to time large-scale fluctuations occur in various parts of the Universe, which increase the organization level (the idea of Boltzmann) [28]. This assumption requires eternal past, since the probability of giant fluctuations is extremely low and they could have hardly occurred in 14 billion years. If we choose initial state after the Big Bang, it is necessary to explain, where the original order had arisen, which set the direction of the arrow of time. However, gravity is the very factor of the order in the initial conditions. The initial state of equilibrium after the Big Bang cannot be same equilibrium in the presence of gravitational interaction, which makes the elements pull up together to form complex structures. In this case, one has to think that time is somehow connected with gravity and, perhaps, is derived from it (in a certain way the GR confirms this). If so, we should clarify what exactly the gravity is. If it is treated in the sense of the GR, as the 
space-time curvature, time is excluded again. It is possible to consider gravity as a result of real carrier particles (gravitons) actions, as the superstring theory, for example, has it and predicts their existence. In this case, time will have to be connected not only to the force of gravity, but, apparently, to repulsive gravity, the cosmological constant, too.

Another modern concept which eliminates time views the Universe as a hologram. This approach has emerged from the black hole studies begun by Jacob Bekenstein [4] and Stephen Hawking [18], was continued by Gerard 't Hooft [43, p. 621] and Leornard Susskind [44] and was completed by Edward Witten [48] and Juan Maldacena [19]. T'Hooft and Saaskind showed that all the information about any object can be recorded on its surface area, i.e. the information within the area is always smaller than the surface. This suggests that the arena of physical laws is just the border and the observed three-dimensional reality is a holographic projection ${ }^{8}$. Maldacena, a string theorist, following the principles of Witten, has revealed the possibility of a dual description of reality. His string theory (the strings in the beam) is identical to the quantum field theory. This has become possible because the same mathematical vocabulary is used to describe what is happening inside the Maldacena's world ${ }^{9}$ and on the border of this world (the actual quantum field theory). Thus, both theories are essentially the same, but they describe reality from different perspectives. The essence of the concept is that you can describe what is happening inside by what is happening outside, on the border area. For example, it may mean that a black hole is a holographic projection of gas on its surface. Then black holes may appear to be quite trivial objects ${ }^{10}$. Ultimately, the universe can be described as a hologram, i.e., as a projection from a distant flat surface.

What is the role of time in such a model of describing reality? Should we consider that time is also a projection (and if we consider time to be a derivative from the laws of physics and arising from them, rather than preceding them, a projection of what is time then)? In the spirit of Plato and Platonists one can say that it is "the projection of eternity", but from the viewpoint of physics, the answer, of course, is not concrete enough. Probably, it would be right to say that there is no time on the surface, time is just a property of three-dimensional projection. The projection is moving, and here, as Heraclitus put it, "everything flows, everything changes", but the boundary surface remains unchanged (because it is atemporal). This means that time is not fundamental and is derived from something else that is encoded on the remote surface. And currently it is not clear what could that something be.

Another option, which is in a better compliance with the string theory, is that time (and space) is a predetermined pattern, a stage for events. One version of a cyclic universe (or multiverse), which has been proposed in the superstring theory, considers time precisely this way. In the model, proposed by Paul Steinhardt and his colleagues [25], our universe has been discussed as a three-dimensional brane, located in the space of a higher dimension. From time to time, a collision with other brane-universe ${ }^{11}$ may occur, which means end of these universes and arising of new ones.

Another cyclic theory within more classical beliefs has been proposed by Penrose [36]. His concept suggests that a new Universe is the result of fluctuation (in fact another Big Bang), which is inevitable an infinite time later after reaching the thermal equilibrium. When this latter is accomplished, later universe becomes indistinguishable from the earlier one. Thus, the end becomes a new beginning. Interestingly, this theory, despite of the obviously strange need in expiration of infinite time, is able to be verified. The detection of gravitational waves and concentric circles from the collision of several black hole pairs may speak in its favor. There is evidence that such data have been obtained [14].

But any cyclic model, no matter what it is based on, requires the presence of predetermined time outside of universe, which is not going to arise and die along with the universe. Indeed, the cyclic scenario makes no sense when time appears with the emergence of the universe. How can one claim the previous existence of universes, if their time had disappeared together with them? If there are timeless intervals between universes (which fact is absurd of itself), we cannot use terms "before", "was", etc. 
Such scenarios only complicate the problem of time. Time here appears to be a certain fundamental value, which is wittingly impossible to perceive, as it is placed outside the world. The same applies to the different concepts of multiverse [23]. If we exclude the external time and leave only the proper time of each world, the question arises: how do the worlds relate to each other in time? The theory of eternal inflation [17] raises another question: is there time in the inflaton field or Plato's eternity reigns over it and time appears only together with the worlds, in "bubble" universes? In this case, timeless would separate worlds, which cannot be comparable over time and we can neither say that the worlds have different time, nor that they have the same one. This issue also does not lose its relevance in the case of Everett's many-worlds interpretation, string landscape and some other multiverse theories.

The interpretation of time in quantum field theory is also quite special. The Schrödinger equation describes the wave function of the particle before it is measured, at the very moment of measurement the wave function collapses and the macrocosm now dictates its rules to situation.

Thus, time plays a key role in the act of measurement. Measurement changes the future. Before the measurement the past of a particle is blurry (it may be anywhere with a number of the most likely positions and, more precisely, it may by anywhere at the same time if hidden variables are not allowed). At the moment of measurement the particle is detected somewhere and the rules of a microcosm no longer work for it. If the measurement had not been carried out, the particle would have been further described by a wave function. In this situation the moment of measurement has special, determining the future powers - this is the very moment of present, which separates the future from the past. On its only basis we cannot reconstruct the past and are able to only statistically predict the future. The past and the future are always blurred, only the present exists.

From the viewpoint of the hidden variable theories [6] the act of measurement itself is nothing special. It just allows detecting the previously unknown location of a particle. The concept of Hugh Everett [15] suggests that measurement also does not have any special status and appears to be one of the possible realizations in parallel universes. Most of the other interpretations of quantum mechanics also avoid the problem of measurement [5].

The standard quantum field theory, however, has to recognize a selected moment in time (it is important that it is chosen by the observer, i.e. a macrocosm object). In a microcosm time reveals itself only in interaction with the macrocosm, when the observer comes to intervene. So, does this mean that the concept of time is only meaningful in macrocosm and it makes no sense in microcosm? This point of view has existed for quite a long time. In fact, the classical representations of space and time at the scale of Planckian quantities are probably pointless. However, for lack of better options physicists and mathematicians have to use traditional systems of coordinates and clock. To be more precise, the classic time and space makes sense, but only at the moment of transition from micro- to macrolevel.

As an alternative to the general relativity theory a number of physicists [16], [3] suggest Shape Dynamics. The fundamental difference of the Shape Dynamics theory is that time here is considered to be universal, while space is relative. This means that there is a distinguished observer and, accordingly, allotted time. The relativity of space means that in different parts of the Universe the size of similar objects may be different or more precisely, the concept of size over long distances has no independent meaning, just as the concept of simultaneity of events in the theory of relativity. Global time and simultaneous observation are possible, because, for example, a universal frame of reference had been chosen - the microwave background radiation. The observers will register its one and the same temperature in all directions of the universe, so there are separated observers (which fact, however, brings asymmetry into GRT). It is important that Shape Dynamics is a dual description of GRT; the relativity of time is replaced by the relativity of space, two theories are equivalent to each other.

The postulation of initial to physics laws absolute time leads to curious consequences: laws can change over time. I.e. laws turn out to be not invariable, not so fundamentally basic for time determination, but they occur in time themselves. This is certainly an interesting approach that 
allows a fresh look at the evolution of the Universe. But this approach leaves the essence of time unexplained. On the contrary, the assumption of changing the laws of physics over time requires the mechanism of this change to be explained, so there must be some principle of the laws evolution over time. There is also another possibility: to say that time is ultimate and it exists for no particular reason, but it is a cognitive dead-end.

Thus, Shape Dynamics is trying to solve the problem of choosing initial conditions by introducing asymmetric solutions in time. Time exists, it is unchangeable, but things change over time, including the laws of physics (perhaps, new laws appear).

Another important feature of Shape Dynamics (which makes it even possible to reconstruct events in the past) is that it is consistent with the theory of hidden variables in quantum mechanics, i.e. with the idea that all particles have a position and velocity at any point in time (such an assumption suggests the need for a distinguished observer). This is exactly what Einstein has demanded from the theory and what became possible only under the dual description of his theory.

\section{Conclusion}

Alongside with the growth of scientific knowledge the intellectual intuition adapts to the formulation of new concepts and modernization of old ones (inverse relation is also true). With the incipiency of new physical theories (experimental confirmation is optional here) an updating of traditional question formulation is often required. For example, the theory of relativity introduces the relativity of simultaneity concept - a fundamentally new step in science, which requires a rethinking of the category of time (that ends up in GRT with eliminating of time). Another example is the quantum field theory, where the time as the distinction of past and future occurs only during the transition from micro- to macrolevel. The holographic principle, which has grown out of the possibility of a dual description of physical systems, offers again a completely new way of time understanding: time as projection (or requires the acceptance of two time origins). The multiverse (and the cyclic Universe) concepts raise a fundamental question on global time - whether in each universe its separate time exists or whether time is common to all of them.

Finally, the question of time dimension is a really new issue in the problem. Time has traditionally been considered as either a circle (cyclical) or as an arrow. If time can be of great dimension, as the folded spaces in superstring theory, it is most likely confirming the validity of the concept of time geometrization and shows a lack of grounds for search of essentially temporary categories. I.e., it is possible that time is a variety of space.

As it has become possible to find out, most of related to time issues have not been significantly changed, comparing to earlier attempts of its philosophical interpretation.

Considering that, the key stated at the beginning question - if the time exists - has no positive solutions. In those theories, where the answer is positive, it is fundamental in the sense that it is initial. This option cannot still define that the time is, but on the contrary, takes a step back in an attempt to answer this question. After declaring something as initial, we can continue considering it only in the spirit of negative theology, since nothing becomes the cause of it.

In the concepts, where time appears, it is possible to consider it existing; but so far the only variant for its explanation is the space. However, understanding of time (not at all new) as a movement in space (the sequence of events in space or even a special kind of space) sort of eliminates the time from the concept of time. These approaches deny their own temporal time specifics (which may become true).

It is likely that the part of the problem lies in the inability of intellectual intuition to exceed the bounds of the ordinary idea of time and the longing for fitting it into the familiar pattern of macrocosm. In this case, an effective way to overcome this would be a formulation of new concepts of time and space on the basis of the experimental results and mathematical description, which severs the tradition of thinking that produced formulated by Zeno problems. In a sense, the essence 
of his paradoxes specifically points to a disparity between the intellectual intuition and physical reality, rather than to the impossibility of movement.

\section{Acknowledgement}

This work is an output of a research project implemented as part of the Basic Research Program at the National Research University Higher School of Economics (HSE).

\section{References}

1. Aristotle. The Physics. Books I-IV. Harvard University Press, Cambridge, 1957.

2. Augustine. Confessions. Oxford University Press, Oxford, 2009.

3. Barbour, J., Koslowski, T., and Mercati, F. The solution to the problem of time in shape dynamics. Classical and Quantum Gravity, 2014, vol. 31, no 15, doi: 10.1088/02649381/31/15/155001.

4. Bekenstein, J. Black holes and entropy. Physical Review, 1976, D. 7, pp. 2333-2346.

5. Bell, J. S. Speakable and unspeakable in quantum mechanics. Cambridge University Press, Cambridge, 1987.

6. Bohm, D. A Suggested Interpretation of the Quantum Theory in Terms of 'Hidden' Variables', I and II. In. Quantum theory and measurement. J. A. Wheeler, and W. H. Zurek (eds.). Princeton University Press, Princeton, 1983, pp. 369-397.

7. Boltzmann, L. Further Studies on the Thermal Equilibrium of Gas Molecules. In. Kinetic Theory of Gases: An Anthology of Classic Papers With Historical Commentary. S. G. Brush, and N. S. Hall (eds.). Imperial College Press: London, 2003, pp. 262-349.

8. Bussières, Clausen, C., Tiranov, A., Korzh, B., Verma, V., Sae Woo Nam, Marsili, F., Ferrier, A., Goldner, P., Herrmann, H., Silberhorn, C., Sohler, W., Afzelius, M., and Gisin, N. Quantum Teleportation from a Telecom-Wavelength Photon to a Solid-State Quantum Memory. Nature Photonics, 2014, no 8(10), pp. 775-778.

9. Chanda, P.-W., and Smolin, L. Disordered Locality as an Explanation for the Dark Energy. Physical Review, 2009, D 80, 063505, doi: 10.1103/PhysRevD.80.063505.

10. Clausius, R. Übereineveränderte Form des zweiten Hauptsatzes der mechanischen Wärmetheorie. Annalen der Physik, 1854, no 12, pp. 481-506.

11. Einstein, A. Zur Elektrodynamik bewegter Körper. Annalen der Physik, 1905, vol. 322, Issue 10, pp. 891-921.

12. Einstein, A. Relativity: The Special and the General Theory. Routledge, London, 1954.

13. Einstein, A., Podolsky, B., and Rosen, N. Can quantum-mechanical description of physical reality be considered complete? Physical Review, 1935, vol. 47, pp. 777-780.

14. Eriksen, H. K., and Wehus, I.K.A search for concentric circles in the 7 year wilkinson microwave anisotropy probe temperature sky maps. The Astrophysical Journal Letters, 2011. vol. 733, no. 2. doi: 10.1088/2041-8205/733/2/L29.

15. Everett, H. 'Relative state' formulation of quantum mechanics. Reviews of Modern Physics, 1957, vol. 29, no 3, pp. 454-462.

16. Gomes, H., Gryb, H., and Koslowski, T. Einstein gravity as a 3D conformally invariant theory. Classical and Quantum Gravity, 2011, vol. 28, no. 4, doi: 10.1088/0264-9381/28/4/045005.

17. Guth, A. The Inflationary Universe: The Quest for a New Theory of Cosmic Origins. Perseus Books, New York, 1997.

18. Hawking, S. W. Black Hole Explosions? Nature, 1974, no. 248 (5443), pp. 30-31.

19. Horowitz, G.T., Maldacena, J., and Strominger, A. Nonextremal Black Hole Microstates and UDuality. Physical Letters B, 1996, no. 383, pp. 151-159.

20. Kaluza, T. Zum Unitätsproblem in der Physik. Sitzungsber. Preuss. Akad. Wiss., Berlin, 1921, pp. 966-972.

21. Kant, I. Critique of Pure Reason. Cambridge University Press, Cambridge, 1998. 
22. Karpenko, I. Question of Consciousness: to Quantum Mechanics for the Answers. Studia Humana, 2014, vol. 3, no. 3, pp. 16-28.

23. Karpenko I. Notion of Space in Some Modern Physics Theories. Jepistemologija i filosofija nauki, 2015, vol. XLV, no. 3, pp. 150-166.

24. Karpenko, A.S. V poiskah real'nosti: ischeznovenie [In Search of Reality: Disappearance]. Filosofija nauki i tehniki, 2015, vol. 20, no. 1, pp. 36-81 (in Russian).

25. Khoury, J., Ovrut, A.B., Steinhardt, P.J., and Turok, N. Ekpyrotic universe: Colliding branes and the origin of the hot big bang. Physical Review, 2001, D 64, doi: http://dx.doi.org/10.1103/PhysRevD.64.123522.

26. Klein, O.Quantentheorie und fünfdimensionale Relativitätstheorie. Zeitschrift für Physik, 1926, A 37 (12), pp. 895-906.

27. Koyre A. Études d'histoire de la pensée philosophique. Librairie Armand Colin, Paris, 1961.

28. Lebowitz, J. L. Boltzmann's Entropy and Time's Arrow. Physics Today, 1993, vol. 46, no 9, pp. 32-38.

29. Leibniz and Clarke: Correspondence. Hackett Publishing, Indianapolis, 2000.

30. Losev, A.F. Istorija antichnoj jestetiki. Pozdnij jellinizm [History of ancient aesthetics. The late Hellenism]. In. Polnoe sobranie sochinenii [Complete Works], A.F. Losev, vol. 3. Folio, Moscow, 2000, pp. 241-528, (in Russian).

31. Mach, E. The Science of Mechanics: A Critical and Historical Account of Its Development. Open Court Publishing Company, Chicago, 1915.

32. Mangabeira, U. R., and Smolin, L. The Singular Universe and the Reality of Time. Cambridge University Press, Cambridge, 2014.

33. Markopoulou, F., and Smolin, L. Disordered Locality in Loop Quantum Gravity States. Classical and Quantum Gravity, 2007, vol. 24, no 15, doi: 10.1088/0264-9381/24/15/003.

34. Minkowski, H. Raum und Zeit. Physikalische Zeitschrift, 1909, no 10, pp. 75-88.

35. Newton, I. Mathematical Principles of Natural Philosophy. University of California Press, Berkeley, 1999.

36. Penrose, R. Cycles of Time: An Extraordinary New View of the Universe. Knopf, New York, 2011.

37. Plato. Timaeus. Hackett Publishing, Indianapolis, 2000.

38. Plotinus. Ennead 3. Harvard University Press, Cambridge, 1967.

39. Poincaré, H. Sur la dynamique de l'électron. Rendiconti del Circolo Matematico di Palermo, 1906, 21, pp. 129-176.

40. Proclus. The Commentaries of Proclus on the Timous of Plato. British Library, London, Historical Print Edition, 2011.

41. Russel, B.A History of Western Philosophy and Its Connection with Political and Social Circumstances from the Earliest Times to the Present Day. Simon and Schuster: New York, 1945.

42. Smolin, L. Time Reborn: From the Crisis in Physics to the Future of the Universe. Houghton Mifflin Harcourt, New York, 2013.

43. Stephens, C. R., 't Hooft, G., and Whiting, B. F. Black Hole Evaporation without Information Loss. Classical and Quantum Gravity, 1994, vol. 11, pp. 621-647.

44. Susskind, L. The World as a Hologram. Journal of Mathematical Physics, 1995, vol. 36, no 11, pp. 6377-6399.

45. Whitrow, G.J. The natural philosophy of time. Oxford University Press, Oxford, 1980.

46. Whitrow, G.J. The Nature of Time. Penguin Books, London, 1976.

47. Whitrow, G.J. What is time? The Classic Account of the Nature of Time. Oxford University Press, Oxford, 2004.

48. Witten, E. Anti-de Sitter Space and Holography. Advances in Theoretical and Mathematical Physics, 1998, no 2, pp. 253-291. 


\section{Notes}

1. This refers not to the fact that the coordinate system is an objective description of space. Concerning objects and phenomena of microcosm the usual concept of distance loses its meaning, but the system of coordinates can be used as a convenient tool.

2. This section covers only amenable to physical interpretation ideas.

3. Descartes, who was Leibniz's predecessor in asserting the relativity of motion, could not, however, draw a conclusion about the relativity of time from that, and Leibniz, asserting the relativity, failed to notice the possibility of the relativity of simultaneity.

4. Interestingly, the assertion of the subjectivity of time (and, more widely, solipsism) does not deny the program of scientific research. In any case, the question remains: why we do we perceive the time in one way, but not another? Why do we imagine (in the case of solipsism) the world as we do, but not otherwise? The laws of physics should be the answer, determining our perception and thinking. Which means, that science is a search for what stands behind the phenomena, whatever they may be.

5. Hereinafter, it is assumed that if something is counter-intuitive, it does not lead to impossibility of something. This only means the inability to perceive the object of controversy at present.

6. If we accept this, it is fair to mention: non-locality exists, but only at the quantum level, as additional dimensions are folded and the macrocosm is local

7. There is no contradiction here. Einstein space is relative - it only means that its properties are determined by massive objects. But it exists and these objects are within this space. In a certain way, this space-time is an arena of actions, a background, just as Newton's space, but with a feedback.

8. There is a great temptation to rethink the theory of Plato's ideas in the light of the holographic principle.

9. Maldacena's universe is different from ours and, strictly speaking, his results are not applicable to our world. But that does not diminish his importance. The very possibility of a dual description, which allows us to understand complex theories with the help of relatively simple ones, is of great value.

10. This raises another important issue. Inside a black hole time and space reverse their roles, movement in time becomes movement in space and vice versa. The fact of the dual description in this case points to the fundamental indistinguishability of space and time.

11. They are unobservable, because the string math allows only closed strings (gravitons), unlike photons, to travel between the two universes. 